\title{
Deformations of calibrated submanifolds with boundary
}

\author{
Alexei Kovalev \\ DPMMS, University of Cambridge
}

\begin{abstract}
We review some results concerning the deformations of calibrated minimal submanifolds which occur in Riemannian manifolds with special holonomy. The calibrated submanifolds are assumed compact with a non-empty boundary which is constrained to move in a particular fixed submanifold. The results extend McLean's deformation theory previously developed for closed compact submanifolds.
\end{abstract}

\section{Preliminaries}

Calibrated submanifolds are a particular type of minimal submanifolds introduced by Harvey and Lawson [HL] as a generalization of complex submanifolds of Kähler manifolds; for a detailed reference, see op.cit. and $[\mathrm{H}]$. Harvey and Lawson [HL also found four new types on calibrations defined on Euclidean spaces and, more generally, on Ricci-flat Riemannian manifolds with reduced holonomy. Further examples of calibrations were subsequently discovered, see [J, §4.3] and references therein.

McLean [McL] studied deformations for the four types of calibrated submanifolds defined in [HL and showed that the deformation problem may be interpreted as a non-linear PDE with Fredholm properties. In some cases, there is a smooth finite-dimensional 'moduli space'. The submanifolds in $\mathrm{McL}$ ] were assumed compact and without boundary. McLean's deformation theory in $\mathrm{McL}$ was later extended by several authors to more general classes of submanifolds. In this paper, we survey the generalizations of McLean's results to compact submanifolds having non-empty boundary. Proofs will at most be briefly sketched or referred to the original papers.

We begin in this section with some key concepts of calibrated geometry and foundational results concerning deformations of compact submanifolds 
(possibly with boundary). The remainder of the paper is organized in four sections dealing with the four types of calibrated submanifolds in [HL, McL], namely special Lagrangian, coassociative, associative and Cayley submanifolds.

Submanifolds are taken to be embedded and connected, for notational convenience (it can be checked that the results extend to immersed submanifolds). Smooth functions and, more generally, sections of vector bundles on (sub)manifolds with boundary are understood as 'smooth up to the boundary', so at each point of the boundary these have one-sided partial derivatives of any order in the inward-pointing normal direction.

\subsection{Calibrations}

Definition 1. Let $(M, g)$ be a Riemannian manifold. For any tangent $k$ plane $V$, i.e. a $k$-dimensional subspace $V$ of a tangent space $T_{x} M$, a choice of orientation on $V$ together with the restriction of $g$ determines a natural volume form on $V$, $\operatorname{vol}_{V} \in \Lambda^{k} V^{*}$.

A differential $k$-form $\phi$ on $M$ is called a calibration if (i) $d \phi=0$ and (ii) for each $x \in M$ and every oriented $k$-dimensional subspace $V \subset T_{x} M$, $\left.\phi\right|_{V}=a \operatorname{vol}_{V}$ for some $a \leq 1$.

An oriented $k$-dimensional submanifold $N$ of $M$ is said to be calibrated by $\phi$ if the pull-back of $\phi$ to $N$ coincides with the Riemannian volume form for the metric on $N$ induced by $g$, i.e. $\left.\phi\right|_{T_{x} N}=\operatorname{vol}_{T_{x} N}$ for each $x \in N$.

The next result shows that calibrated submanifolds are minimal, in fact volume-minimizing (if compact).

Proposition 2. Let $M$ be a Riemannian manifold and let $\phi \in \Omega^{k}(M)$ be a calibration on $M$.

(a) If a closed $k$-dimensional submanifold $X \subset M$ is calibrated by $\phi$, then $X$ is volume-minimizing in its homology class. Moreover, if $Y$ is a volume-minimizing closed $k$-dimensional submanifold of $M$ in the homology class of $X$, then $Y$ is calibrated by $\phi$.

(b) Let $W \subset M$ be a submanifold such that $\left.\phi\right|_{W}=0$. If $X \subset M$ is a calibrated compact $k$-dimensional submanifold with non-empty boundary $\partial X \subset W$, then $X$ is volume-minimizing in the relative homology class $[X] \in H_{k}(M, W ; \mathbb{Z})$. Moreover, if $Y$ is a compact submanifold of $M$ with boundary $\partial Y \subset W$ and $Y$ is volume-minimizing in the relative homology class $[X]$, then $Y$ is calibrated by $\phi$. 
The clause (a) is proved in [HL, Thm. II.4.2] by application of Stokes' theorem. The extension (b) to submanifolds with boundary follows by a similar argument as the hypothesis $\left.\phi\right|_{W}=0$ ensures the vanishing of the additional terms arising from the boundary (cf. [G, pp. 1233-1234]). Suppose that a submanifold $Y$ with boundary in $W$ represents the relative homology class $[X]$. Considering $X$ and $Y$ as chains, we can find a $(k+1)$-dimensional chain $N$ with boundary in $W$ and a $k$-dimensional chain $P$ contained in $W$ so that $Y-X=\partial N+P$. We then obtain

$$
\operatorname{Vol}(Y) \geq \int_{Y} \phi=\int_{X} \phi+\int_{\partial N} \phi+\int_{P} \phi=\operatorname{Vol}(X),
$$

noting in the inequality that $\phi$ is a calibration, then applying Stokes' theorem and taking account of the vanishing of $\phi$ on $W$.

The above argument may be viewed as a generalization of the volumeminimizing property of compact complex submanifolds of Kähler manifolds by application of Wirtinger's inequality. (In a Kähler manifold with Kähler form $\omega$, every $k$-dimensional complex submanifold is calibrated by $\omega^{k} / k !$.)

\subsection{Normal deformations of submanifolds}

McLean's deformation theory $\mathrm{McL}$ ] was originally developed for closed compact submanifolds. The nearby deformations of a given closed submanifold of a Riemannian manifold may be assumed to be normal deformations, defined using the Riemannian exponential map on normal vector fields, i.e. $C^{1}$ sections of the normal bundle of this submanifold.

When a submanifold has a boundary, we wish to consider the deformation problem as an elliptic boundary value problem. We shall in fact require that the boundary moves in a certain fixed submanifold which, following $[\mathrm{Bu}]$, we call a scaffold. In general, we cannot use, as in the case of closed submanifolds, exponential deformations defined using the given metric $g$ on the ambient manifold, since the scaffold may not be preserved under such deformations. We shall define on $M$ a modified metric $\hat{g}$ whose associated exponential map does preserve the scaffold because the scaffold will be totally geodesic with respect to the new metric. (The actual construction of the metric $\hat{g}$ will depend on the considered calibration.)

\section{Proposition 3.}

(a) Let $P$ be a closed submanifold of a Riemannian manifold $M$.

There exist an open subset $V_{P}$ of the normal bundle $N_{P / M}$ of $P$ in $M$, containing the zero section, and a tubular neighbourhood $T_{P}$ of $P$ in $M$, 
such that the exponential map $\left.\exp _{M}\right|_{V_{P}}: V_{P} \rightarrow T_{P}$ is a diffeomorphism onto $T_{P}$.

(b) Let $M$ be a smooth manifold of dimension $n$ and $P \subset M$ a compact submanifold with non-empty boundary $\partial P$. Let $W$ be a submanifold of $M$ with $\partial P \subset W$ and let $\hat{g}$ be a Riemannian metric on $M$ such that $P$ and $W$ meet orthogonally and $W$ is totally geodesic with respect to $\hat{g}$.

There exists an open subset $V_{P}$ of the normal bundle $\hat{N}_{P / M}$ of $P$ in $M$, containing the zero section, and an n-dimensional submanifold $T_{P}$ of $M$ with boundary such that $P \subset T_{P}$ and $\left.\widehat{\exp }_{M}\right|_{V_{P}}: V_{P} \rightarrow T_{P}$ is a diffeomorphism onto $T_{P}$. Furthermore, if a section $\mathbf{v}$ of $\hat{N}_{P / M}$ takes values in $V_{P}$, then $\widehat{\exp }_{M}(\mathbf{v}(x)) \in W$ for all $x \in \partial P$.

The clause (a) is a consequence of the tubular neighbourhood theorem $[\mathrm{L}$, Chapter IV, Thm. 9]. For the extension (b) to submanifolds with boundary and the existence of the adapted metric $\hat{g}$ modifying a given metric on $M$ on an open neighbourhood of $\partial P$, cf. [Bu, Prop. 6] or [KL, Prop. 4.4]. We stress that the new auxiliary metric $\hat{g}$ in (b) is used solely for the purpose of considering the exponential map and applications of the tubular neighbourhood theorem - but not for the minimal or volume-minimizing properties of calibrated submanifolds (for which we continue to use the original metric on $M)$.

By nearby deformations of a compact submanifold $P$ (with or without boundary) we shall mean submanifolds of the form $P_{\mathbf{v}}=\exp _{\mathbf{v}}(P)$, where $\mathbf{v}$ is a $C^{1}$-section of the normal bundle $N_{P / M}$. The section $\mathbf{v}$ is assumed sufficiently small in the $C^{1}$ norm, so that $P_{\mathbf{v}}$ is contained in a tubular neighbourhood $T_{P}$ defined by Proposition 3. We shall call sections of $N_{P / M}$ the normal vector fields on $P$.

We interpret submanifolds as appropriate equivalence classes of the embedding (more generally, immersion) maps and the term 'moduli space of submanifolds' is used below in this sense.

\section{Special Lagrangian submanifolds in Calabi-Yau manifolds}

Let $M$ be a Kähler manifold of complex dimension $m$, with Kähler form $\omega$ and suppose further that the metric on $M$ has holonomy contained in $S U(m)$. Then the canonical bundle of $M$ may be trivialized by a holomor- 
phic $(m, 0)$-form $\Omega$ satisfying

$$
(-1)^{m(m-1) / 2}(i / 2)^{m} \Omega \wedge \bar{\Omega}=\omega^{m} / m !
$$

It also follows that the Kähler metric is Ricci-flat. Conversely, if a Kähler form $\omega$ and a holomorphic $(m, 0)$-form $\Omega$ satisfy (11) on $M$, then this Kähler metric has holonomy in $S U(m)$. We shall call $(M, \omega, \Omega)$ as above a CalabiYau manifold.

The real $m$-form $\operatorname{Re} \Omega$ is a calibration on $M$ (cf. [HL, Thm. III.1.10]) and the submanifolds calibrated by $\operatorname{Re} \Omega$ are called special Lagrangian submanifolds. It will be convenient to use an equivalent definition.

Proposition 4 ([L, Cor. III.1.11]). Let $(M, \omega, \Omega)$ be a Calabi-Yau manifold of complex dimension $m$. A real m-dimensional submanifold $L \subset M$ with some choice of orientation is special Lagrangian if and only if

$$
\left.\omega\right|_{L}=0 \quad \text { and }\left.\quad \operatorname{Im} \Omega\right|_{L}=0 .
$$

The following result about the deformations of compact special Lagrangian submanifolds without boundary is due to McLean.

Theorem 5 ([McL, Thm. 3.6]). Let $M$ be a Calabi-Yau manifold and $L$ a closed special Lagrangian submanifold in $M$. Then the moduli space of nearby special Lagrangian deformations of $L$ is a smooth manifold of dimension the first Betti number $b^{1}(L)$.

The argument of Theorem 5 uses the equivalent definition (2) of special Lagrangian submanifolds in terms of the vanishing of differential forms.

Applying Proposition 3(a) to $L$ we may write any nearby deformation of $L$ as $L_{v}=\exp _{v} L$ for a section $v \in \Gamma\left(N_{L / M}\right)$ of the normal bundle. On the other hand, there is an isometry of vector bundles

$$
\left.\jmath_{L}: \mathbf{v} \in N_{L / M} \rightarrow(\mathbf{v}\lrcorner \omega\right)\left.\right|_{L} \in \Lambda^{1} T^{*} L
$$

defined using the Kähler form on $M$. Thus the nearby deformations of $L$ are equivalently given by 'small' 1 -forms on $L$.

The map

$$
F: \alpha \in \Omega^{1}(L) \rightarrow\left(\exp _{\mathbf{v}}^{*}(\omega), \exp _{\mathbf{v}}^{*}(\operatorname{Im} \Omega)\right) \in \Omega^{2}(L) \oplus \Omega^{m}(L), \quad \mathbf{v}=\jmath_{L}^{-1}(\alpha)
$$

is defined for 'small' $\alpha$, and $F(\alpha)=0$ precisely if $\exp _{\mathbf{v}}(P)$ is a special Lagrangian deformation. 
Proposition 6. Let $L$ be a special Lagrangian submanifold of a Calabi-Yau manifold and $F$ the 'deformation map' defined in (4).

(a) The map $F$ is smooth, with derivative at $\alpha=0$ given by

$$
\left.d F\right|_{0}(\alpha)=(d \alpha, d * \alpha), \quad \alpha \in \Omega^{1}(L) .
$$

(b) If $L$ is a closed submanifold, then there is a neighbourhood $T_{L}$ of the zero 1-form such that the image $F\left(T_{L}\right)$ consists of pairs of exact forms.

Note that in Proposition 6(a) a special Lagrangian $L$ need not be compact.

The nearby special Lagrangian deformations of $L$ correspond to the 1forms $\alpha$ satisfying a non-linear differential equation $F(\alpha)=0$ of first order, with $F(0)=0$. One can show using Hodge theory for a closed manifold $L$ and the implicit function theorem in Banach spaces that the $C^{1}$-small solutions of the special Lagrangian deformation problem may be parameterised by the closed and co-closed 1-forms $\alpha$ on $L$. By Hodge theory again, as $L$ is a closed manifold, the moduli space of special Lagrangian deformations is then locally parameterised by the vector space of harmonic 1-forms on $L$ or, equivalently, by the de Rham cohomology group $H^{1}(L, \mathbb{R})$ of dimension $b^{1}(L)$. Theorem 5 follows.

The constraint (1) determines a holomorphic form $\Omega$ up to a factor $e^{i \theta}$ for some real constant $\theta$ and $\operatorname{Re}\left(e^{i \theta} \Omega\right)$ is also a calibration on $M$. Manifolds calibrated by $\operatorname{Re}\left(e^{i \theta} \Omega\right)$ are called special Lagrangian with phase $\theta$. Every submanifold calibrated by $\operatorname{Re}\left(e^{i \theta} \Omega\right)$ is Lagrangian (with respect to the symplectic form $\omega$ ) and minimal (with respect to the metric on $M$ ). Conversely, it is known that every connected minimal Lagrangian submanifold in a Calabi-Yau manifold is calibrated by $\operatorname{Re}\left(e^{i \theta} \Omega\right)$ for some real constant $\theta$ [HL, cf. Prop. III.2.17].

If $L^{\prime}$ is a minimal Lagrangian deformation of $L$ then by the above $L^{\prime}$ must be calibrated and volume-mininizing. Therefore, $L$ is special Lagrangian by application of Proposition 2(a). It follows that moduli space in Theorem 5 can be equivalently regarded as the space of nearby minimal Lagrangian deformations of $L$. In particular, there is no loss of generality in restricting attention to submanifolds calibrated by $\operatorname{Re} \Omega$, i.e. with $\theta=0$.

Remark. Salur [Sa] extended the result of Theorem 5 to the situation when the almost complex structure on $M$ is not necessarily integrable. More explicitly, $M$ is a Hermitian symplectic $2 m$-manifold with symplectic form $\omega$, the metric on $M$ is Hermitian with respect to an $\omega$-compatible almostcomplex structure and there is a (non-vanishing) complex $(m, 0)$-form $\Omega$ 
on $M$ satisfying (11). The main theorem in $\mathrm{Sa}$ then asserts that the moduli space of nearby special Lagrangian deformations of $L$ with arbitrary phase is smooth with dimension $b^{1}(L)$. In the case when $d \operatorname{Im} \Omega=0$, it can be checked that any special Lagrangian deformations of $L$ necessarily have the same phase as $L$ (and can be obtained essentially by McLean's argument in $[\mathrm{McL}]$ ).

Suppose now that a compact special Lagrangian submanifold $L$ has non-empty boundary $\partial L$. We shall need the following definition from $[\mathrm{Bu}$, p. 1954].

Definition 7. Let $M$ be a Calabi-Yau manifold and let $L \subset M$ be a submanifold with boundary $\partial L$. Denote by $\mathbf{n} \in \Gamma\left(T_{\partial L} L\right)$ the inward unit normal vector field. A scaffold for $L$ is a smooth submanifold $W$ of $M$ with the following properties:

(1) $\partial L \subset W$

(2) $\mathbf{n} \in \Gamma\left(T_{\partial L} W\right)^{\omega}$ (here, $S^{\omega}$ denotes the symplectic orthogonal complement of a subspace $S$ of a symplectic vector space $V$, defined by $S^{\omega} \equiv\{v \in$ $V: \omega(v, s)=0 \forall s \in S\})$;

(3) the bundle $(T W)^{\omega}$ is trivial.

The deformations of special Lagrangian submanifolds with boundary constrained to be in a fixed scaffold were studied by Butscher who proved the following.

Theorem 8 ([ $\mathrm{Bu}$, Main Theorem $])$. Let $L$ be a compact special Lagrangian submanifold of a Calabi-Yau manifold $M$ with non-empty boundary $\partial L$ and let $W$ be a symplectic, codimension two scaffold for $L$. Then the moduli space of nearby minimal Lagrangian deformations of $L$ with boundary on $W$ is finite dimensional and is locally parameterised by the vector space of closed co-closed 1-forms on L satisfying Neumann boundary conditions

$$
\left.\mathcal{H}_{\mathbf{n}}^{1}(L)=\left.\left\{\alpha \in \Omega^{1}(L): d \alpha=0, d^{*} \alpha=0,(\mathbf{n}\lrcorner \alpha\right)\right|_{\partial L}=0\right\} .
$$

Theorem 8 allows special Lagrangian deformations of $L$ with arbitrary phase $\theta$ and the proof uses an extended version of the deformation map including $\theta$ as an additional variable. The deformation map also requires a construction of an auxiliary metric $\hat{g}$ so that $W$ is totally geodesic for $\hat{g}$ and the appropriate version of the tubular neighbourhood theorem (Proposition $3(b)$ ). (The condition (3) in the definition of the scaffold is used in the construction of $\hat{g}$.) The argument then proceeds by appealing to the implicit function theorem in a similar manner to the deformation problem for 
closed submanifolds. This requires a version of Hodge theory for compact manifolds with boundary [Sch] to identify appropriate Banach subspaces of forms so that the linearization of the deformation map be surjective.

The vector space $\mathcal{H}_{\mathbf{n}}^{1}(L)$ in Theorem 8 is naturally isomorphic to the real cohomology group $H^{1}(L, \mathbb{R})$ and thus has dimension $b^{1}(L)$ by Hodge theory for manifolds with boundary, see [CDGM, p. 927].

Observe that the condition (2) in the definition of a scaffold means that $J \mathbf{n}$ is perpendicular to $W$. This will be automatically satisfied when $W$ is a complex submanifold of positive codimension in $M$, so the tangent spaces of $W$ are invariant under $J$. In this case, we also have $\left.\Omega\right|_{W}=0$. Then, applying Proposition 2(b), we obtain that for each special Lagrangian $L$ with boundary in $W$ the minimal Lagrangian deformations of $L$ with boundary confined to $W$ will actually be special Lagrangian. We thus obtain a variant of Butscher's result for the space of special Lagrangian deformations.

Corollary 9. Let $L$ be a compact special Lagrangian submanifold of a Calabi-Yau manifold $M$ with non-empty boundary $\partial L$. Let $W$ be a complex codimension one submanifold of $M$ with trivial normal bundle and with $\partial L \subset W$ (in particular, $W$ is a scaffold for $L$ ). Then the moduli space of nearby special Lagrangian deformations of $L$ is a smooth manifold of dimension $b^{1}(L)$.

\section{Coassociative submanifolds in $G_{2}$-manifolds}

The two calibrations considered in this and the next section are defined on 7dimensional manifolds with a torsion-free $G_{2}$-structure. We shall first briefly recall some key definitions. The readers are referred to [HL], [J, Ch. 11,12] and the article by Karigiannis in this volume for a more detailed account of $G_{2}$-structures and the related calibrations.

The group $G_{2}$ can be defined, following $[\mathrm{Br}$, as the stabilizer, in the standard action of $G L(7, \mathbb{R})$ on $\Lambda^{3}\left(\mathbb{R}^{7}\right)^{*}$, of the 3 -form

$$
\varphi_{0}=d x^{123}+d x^{145}+d x^{167}+d x^{246}-d x^{257}-d x^{347}-d x^{356},
$$

where $\mathrm{d} x^{123}=d x^{1} \wedge d x^{2} \wedge d x^{3}$ and so on, with $x^{1}, \ldots, x^{7}$ the usual coordinates on the Euclidean $\mathbb{R}^{7}$. The 3 -form in (5) encodes the cross-product defined by considering $\mathbb{R}^{7}$ as pure imaginary octonions and setting $\varphi_{0}(a, b, c)=$ $\langle a \times b, c\rangle$. The group $G_{2}$ is a 14-dimensional Lie group and a subgroup of $S O(7)$.

The Hodge dual of $\varphi_{0}$ is a 4 -form given by:

$$
* \varphi_{0}=d x_{4567}+d x_{2367}+d x_{2345}+d x_{1357}-d x_{1346}-d x_{1256}-d x_{1247} \text {. }
$$


Let $M$ be a 7-dimensional manifold. We say that a differential 3-form $\varphi$ on $M$ is positive, or is a $G_{2}$ 3-form, if for each $p \in M$ there is a linear isomorphism $\iota_{p}: \mathbb{R}^{7} \rightarrow T_{p} M$ with $\iota_{p}^{*}(\varphi(p))=\varphi_{0}$, where $\varphi_{0}$ is given in (5). Every $G_{2}$-structure on $M$ can be induced by a positive 3 -form $\varphi$ and we shall, slightly informally, say that $\varphi$ is a $G_{2}$-structure. As $G_{2} \subset S O(7)$, every $G_{2}$-structure $\varphi$ induces on $M$ a metric $g(\varphi)$ and orientation and thus also a Hodge star $*_{\varphi}$.

The intrinsic torsion of a $G_{2}$-structure $\varphi$ on $M$ vanishes precisely when $d \varphi=0$ and $d *_{\varphi} \varphi=0$ ([FG]). In this case, we call $(M, \varphi)$ a $G_{2}$-manifold.

The 4 -form $*_{\varphi} \varphi$ defines on each $G_{2}$-manifold $M$ a calibration (cf. [HL, $\S[V .1 . B])$. In fact, the results in this section only require that the $G_{2} 3$ form be closed $d \varphi=0$. We say that an oriented 4-dimensional submanifold $X \subset M$ is a coassociative submanifold if the equality $\left.*_{\varphi} \varphi\right|_{X}=\operatorname{vol}_{X}$ is attained. If in addition $d *_{\varphi} \varphi=0$ holds, then $X$ is calibrated by $*_{\varphi} \varphi$ and we call $X$ a coassociative calibrated submanifold.

The following equivalent definition of coassociative submanifolds will be useful.

Proposition 10 (cf. [HL, Cor. IV.1.20]). For an orientable 4-dimensional submanifold $X$ of a 7-manifold $M$ with a $G_{2}$-structure $\varphi \in \Omega_{+}^{3}(M)$, the equality $\left.*_{\varphi} \varphi\right|_{X}=\operatorname{vol}_{X}$ holds for some orientation of $X$ if and only if $\left.\varphi\right|_{X}=0$.

Let $\varphi$ be a closed $G_{2}$ 3-form on $M$ and let a submanifold $X \subset M$ be coassociative. Then the infinitesimal deformations of $X$ can equivalently be given by self-dual 2-forms on $X$ via an isometry of vector bundles (cf. [McL, Proposition 4.2])

$$
\left.\jmath_{X}: \mathbf{v} \in N_{X / M} \rightarrow(\mathbf{v}\lrcorner \varphi\right)\left.\right|_{X} \in \Lambda_{+}^{2} T^{*} X
$$

where $\Lambda_{+}^{2} T^{*} X$ denotes the bundle of self-dual 2-forms. (The corresponding statements in McL use anti-self-dual 2-forms because McLean uses a different sign convention for the $G_{2} 3$-form.) The map

$$
F: \alpha \in \Omega_{+}^{2}(X) \rightarrow \exp _{\mathbf{v}}^{*}(\varphi) \in \Omega^{3}(X), \quad \mathbf{v}=\jmath_{X}^{-1}(\alpha),
$$

is defined for 'small' $\alpha$, and $F(\alpha)=0$ precisely if $\exp _{\mathbf{v}}(X)$ is a coassociative deformation.

The next theorem summarizes the results obtained by McLean about the deformations of closed coassociative submanifolds.

Theorem 11 (cf. [McL, Thm. 4.5], [JS, Thm. 2.5]). Let $M$ be a Y-manifold with a closed $G_{2}$-structure $\varphi$ and $X \subset M$ a coassociative submanifold (not necessarily closed). 
(a) Then for each $\alpha \in \Omega_{+}^{2}(X)$, one has $\left.d F\right|_{0}(\alpha)=d \alpha$ and the 3-form $F(\alpha)$ (if defined) is exact.

(b) If, in addition, $X$ is compact and without boundary then every closed self-dual 2-form $\alpha$ on $X$ arises as $\alpha=\jmath_{X}(\mathbf{v})$, for some normal vector field $\mathbf{v}$ tangent to a smooth 1-parameter family of coassociative submanifolds containing $X$. Thus, in this case, the space of nearby coassociative deformations of $X$ is a smooth manifold parameterized by the space $\mathcal{H}_{+}^{2}(X)$ of closed self-dual 2-forms on $X$.

Remark. Self-dual 2-forms on a compact manifold without boundary are closed precisely if they are harmonic. By Hodge theory, the dimension of $\mathcal{H}_{+}^{2}(X)$ is therefore equal to the dimension $b_{+}^{2}(X)$ of a maximal positive subspace for the intersection form on $X$. It is thus a topological invariant.

The hypotheses of Theorem 11 do not include the co-closed condition $d *_{\varphi} \varphi=0$. In fact, the argument in $[\mathrm{McL}]$ constructs a smooth moduli space of closed submanifolds $X$ satisfying $\left.\varphi\right|_{X}=0$, for a closed $G_{2}$-structure $\varphi$.

There is a certain analogy between McLean's deformation theory of closed coassociative submanifolds of $G_{2}$-manifolds and closed special Lagrangian submanifolds of Calabi-Yau manifolds. In both cases, the respective submanifolds are calibrated and minimal and have an equivalent definition in terms of the vanishing of appropriate real differential forms on the ambient manifold. The deformation theory is 'unobstructed' and there is a smooth finite-dimensional moduli space, locally parameterised by some finite-dimensional space of harmonic forms on the submanifold with the dimension a topological invariant obtained by Hodge theory.

On the other hand, when the submanifold has a boundary the deformation theories become rather different. As we explain below, following $\mathrm{KL}$, the deformation problem for compact coassociative submanifolds with boundary cannot possibly be set as a boundary value problem of first order with standard Dirichlet or Neumann boundary conditions. Instead the deformation problem will be 'embedded' in a boundary value elliptic problem of second order.

A suitable choice of boundary considerations is again facilitated by the concept of (a coassociative version of) a scaffold which we now define. By way of preparation, we consider an orientable 6-dimensional submanifold $S$ in a 7 -manifold $M$ with a closed $G_{2} 3$-form $\varphi$ on $M$. The normal bundle of $S$ is trivial and there is a 'tubular neighbourhood' $T_{S}$ of $S$ diffeomorphic to $S \times\{-\varepsilon<s<\varepsilon\}$, such that $S$ corresponds to $\{s=0\}$ and $\mathbf{n}=\frac{\partial}{\partial s}$ is a unit vector field on $T_{S}$ with $\left.\mathbf{n}\right|_{S}$ orthogonal to $S$ in the metric $g(\varphi)$. More 
precisely, we consider a coassociative submanifold with (compact) boundary contained in $S$ and then the required $T_{S}$ exists after shrinking $S$ to some neighbourhood of this boundary. We can write

$$
\left.\varphi\right|_{T_{S}}=\omega_{s} \wedge d s+\Upsilon_{s},
$$

for some 1-parameter families of 2-forms $\omega_{s}$ and 3 -forms $\Upsilon_{s}$ on $S$.

The forms $\left.\omega_{0}=\left(\mathbf{n}_{S}\right\lrcorner \varphi\right)\left.\right|_{S}$ and $\Upsilon_{0}=\left.\varphi\right|_{S}$ together define an $S U(3)$ structure on $S$, in general with torsion. This can be seen point-wise, by a consideration similar to $G_{2}$-structures earlier in this section, from the property that the simultaneous stabilizer of $\omega_{0}$ and $\Upsilon_{0}$ in the action of $G L\left(T_{p} S\right)$ at each $p \in S$ is isomorphic to $S U(3)$. In particular, $\omega_{0}^{3}$ defines an orientation on $S$.

Definition 12. An orientable 6-dimensional submanifold $S$ is a symplectic submanifold of a 7-manifold $(M, \varphi)$ with a closed $G_{2}$-structure if $d_{S} \omega_{0}=0$, where $\omega_{0}$ is as defined above and $d_{S}$ is the exterior derivative on $S$.

A 3-dimensional submanifold $L \subset S$ of a symplectic submanifold $S \subset M$ is said to be special Lagrangian if $\left.\omega_{0}\right|_{L}=0$ and $\left.\varphi\right|_{L}=0$.

Every $S$ in the above definition has a Hermitian symplectic structure compatible with the $S U(3)$-structure induced from $M$. In particular, a non-vanishing (3,0)-form on $S$ is obtained as $\Omega_{0}={ }^{*} \Upsilon \Upsilon-i \Upsilon$, where the 6dimensional Hodge start $*_{S}$ is taken with respect to the orientation $\omega_{0}^{3}$ and the induced metric from $M$. As noted in the previous section, McLean's theory remains valid and any closed special Lagrangian $L \subset S$ has a smooth moduli space of dimension $b^{1}(L)$ of nearby special Lagrangian deformations.

Definition 13. Let $(M, \varphi)$ be a 7 -manifold with a closed $G_{2}$-structure and $X \subset M$ a coassociative submanifold with boundary $\partial X$. We say that an orientable 6-dimensional submanifold $S$ of $M$ is a scaffold for $X$ if

(a) $X$ meets $S$ orthogonally, i.e. $\partial X \subset S$ and the normal vectors to $S$ at $\partial X$ are tangent to $X,\left.\mathbf{n} \in N_{S / M}\right|_{\partial X}$, and

(b) $S$ is a symplectic submanifold of $(M, \varphi)$.

One notable property of a scaffold $S$ in Definition 13 is that for each coassociative $X$ meeting $S$ orthogonally, the intersection $L=X \cap S$ is special Lagrangian in $S$.

The infinitesimal deformations of compact coassociative $X$ with boundary in a fixed submanifold $S$ correspond via (6) to a subspace of self-dual 
2-forms on $X$ satisfying boundary conditions. We can write the restriction of any 2-form $\alpha$ on $X$ to a collar neighbourhood $C_{\partial X}=T_{S} \cap X$ of the boundary as $\tilde{\alpha}=\alpha_{\tau}+\alpha_{\nu} \wedge d s$. The Dirichlet and Neumann boundary conditions for $\alpha$ are then given by, respectively, $\alpha_{\tau}=0$ and $\alpha_{\nu}=0$. When $\alpha$ is self-dual, the two conditions are equivalent and force $\alpha$ and the corresponding normal vector field $\jmath_{X}^{-1}(\alpha)$ to vanish at each point of $\partial X$. However, if $d \alpha=0$ and $\alpha$ vanishes on the boundary then $\alpha=0$ by [CDGM, Lemma 2]. This may be understood as an extension of [HL, Thm. IV.4.3], which states that there is a locally unique coassociative submanifold containing any real analytic 3 -dimensional submanifold upon which $\varphi$ vanishes.

It turns our that a suitable choice of the infinitesimal deformations with boundary condition is given by the following subspace of self-dual 2-forms

$$
\left.\left.\Omega_{+}^{2}(X)_{\mathrm{bc}}=\left\{\alpha \in \Omega_{+}^{2}(X): \mathbf{n}\right\lrcorner d \alpha=0 \text { and } d_{\partial X}(\mathbf{n}\lrcorner \alpha\right)=0 \text { on } \partial X\right\} .
$$

The boundary condition $\mathbf{n}\lrcorner d \alpha=0$ ensures that every harmonic form in $\Omega_{+}^{2}(X)_{\mathrm{bc}}$ is closed and the boundary condition $\left.d_{\partial X}(\mathbf{n}\lrcorner \alpha\right)=d_{\partial X} \alpha_{\nu}=0$ means that the boundary $\partial X$ will only move in the space of special Lagrangians in $S$.

The subspace of $\Omega_{+}^{2}(X)_{\mathrm{bc}}$ of the coassociative infinitesimal deformations is given by the harmonic (or closed) forms $\left(\mathcal{H}_{+}^{2}\right)_{\mathrm{bc}}$. It has a finite dimension $\leq b^{1}(\partial X)$ and our next theorem asserts that elements of $\left(\mathcal{H}_{+}^{2}\right)_{\mathrm{bc}}$ 'integrate' to actual coassociative deformations of $X$.

Theorem 14 ([KL, Thm. 1.1]). Suppose that $M$ is a 7-manifold with a $\mathrm{G}_{2}$-structure given by a closed 3-form. The moduli space of compact coassociative local deformations of $X$ in $M$ with boundary $\partial X$ in a scaffold $S$ is a finite-dimensional smooth manifold parameterized by $\left(\mathcal{H}_{+}^{2}\right)_{b c}$. The dimension of this moduli space is not greater than $b^{1}(\partial X)$.

Here is an example when strict inequality $\operatorname{dim}\left(\mathcal{H}_{+}^{2}\right)_{\mathrm{bc}}<b^{1}(\partial X)$ occurs.

Example 15 ([KL, p. 72]). A Kähler complex 3-fold $(Z, \omega)$ is called almost Calabi-Yau if it admits a nowhere vanishing holomorphic $(3,0)$-form $\Omega$. Then the 7-manifold $M=Z \times S^{1}$ has a closed $G_{2}$-structure $\omega \wedge d \theta+\operatorname{Re} \Omega$, where $\theta$ is a coordinate on $S^{1}$. Let $X=L \times S^{1} \subset M$ be a compact coassociative 4 -fold. Then $L$ is special Lagrangian in $Z$. We can think of $X$ as an embedding of a manifold $L \times[0,1]$ whose two boundary components, $L \times\{0\}$ and $L \times\{1\}$, are mapped to $L$ in $Z$. (If $Z$ is Calabi-Yau, i.e. if (1) also holds, then $X$ is a calibrated coassociative.) It is not difficult to see that $Z \times$ pt is a scaffold for $X$. Theorem 14 gives us that $X$ has a smooth moduli space of coassociative deformations with dimension $\leq 2 b^{1}(L)$. 
Let $\alpha \in \Omega_{+}^{2}(X)$. Then $\alpha=\xi_{\theta} \wedge d \theta+*_{L} \xi_{\theta}$, for some path of 1-forms $\xi_{\theta}$ on L. It follows from [Sch, Thm. 3.4.10] that a harmonic self-dual 2form on $X$ is uniquely determined by its values $\xi_{0}, \xi_{1}$ on the boundary. The subspace of harmonic $\alpha \in \Omega_{+}^{2}(X)$ such that $\xi_{0}$ and $\xi_{1}$ are harmonic on $L$ has dimension $2 b^{1}(L)$ and corresponds precisely to the paths $\xi_{\theta}=(1-\theta) \xi_{0}+\theta \xi_{1}$. On the other hand, $\alpha \in\left(\mathcal{H}_{+}^{2}\right)_{\mathrm{bc}}$ if and only if $\alpha$ is harmonic and $\partial \xi_{\theta} / \partial \theta=0$, so $\xi_{0}=\xi_{1}$. Thus $\operatorname{dim}\left(\mathcal{H}_{+}^{2}\right)_{\mathrm{bc}}=b^{1}(L)<b^{1}((L \times\{0\}) \sqcup(L \times\{1\}))$ in this example.

This can also be seen geometrically. If the deformations of the aforementioned two boundary components coincide in $Z \times$ pt then, by taking a product with $S^{1}$, we obtain a coassociative deformation of $X=L \times S^{1}$ defining a point in the moduli space in Theorem 14. On the other hand, if a coassociative deformation $\tilde{X}$ of $X$ is such that the deformations $\tilde{L}_{0}$ and $\tilde{L}_{1}$ of $L \times\{0\}$ and $L \times\{1\}$ are special Lagrangian but distinct then $\tilde{X}$ and $\tilde{L}_{0} \times S^{1}$ are two distinct coassociative 4 -folds intersecting in a real analytic 3 -fold on which $\varphi$ vanishes, which contradicts [HL, Thm. IV.4.3]. Therefore, the moduli space in this example is identified with special Lagrangian deformations of $L$ in the almost Calabi-Yau manifold $Z$. As we noted earlier, these deformations have a smooth moduli space of dimension $b^{1}(L)$.

\section{Associative submanifolds in $G_{2}$-manifolds}

Let $M$ be again a 7 -dimensional manifold with a $G_{2}$-structure given by a positive 3 -form $\varphi$, as defined in the previous section. If $d \varphi=0$ then $\varphi$ is a calibration on $M$ as $\left.\varphi_{p}\right|_{V} \leq \operatorname{vol}_{V}$ for each oriented 3-plane in $T_{p} M$ (cf. [HL, $§ I V .1 . A])$. The equality is attained precisely when $V$ is an associative subspace of $T_{p} M$, i.e. is closed under the cross-product on $T_{p} M$ induced by the $G_{2}$-structure $\varphi$. (The corresponding subalgebra $(V, \times)$ is isomorphic to $\mathbb{R}^{3}$ with the standard vector product.)

The deformation theory discussed in this section does not always require the $G_{2}$ form $\varphi$ to be closed. Similarly to the discussion of coassociative submanifolds in the previous section, we shall define the term associative submanifold $Y$ in $(M, \varphi)$ for an arbitrary $G_{2}$ structure $\varphi$, meaning a 3dimensional submanifold $Y$ satisfying $\left.\varphi\right|_{Y}=$ vol $Y$. If also the $G_{2}$-structure is closed $d \varphi=0$, then we shall call $Y$ an associative calibrated submanifold; indeed, in this case $Y$ is calibrated by $\varphi$.

McLean [McL] studied the deformation theory of closed associative calibrated submanifolds in $G_{2}$-manifolds. His results were later extended by Akbulut and Salur [AS] to arbitrary $G_{2}$-structures on 7 -manifolds. We as- 
sume a torsion-free $G_{2}$-structure to simplify some details.

As in the previous sections, it is useful to first note that associative submanifolds of $M$ can be equivalently defined by the vanishing of an appropriate differential form on $M$. In the present case, it is a 3 -form $\chi$ with values in $T M$,

$$
\left.\chi=\sum_{k=1}^{7}\left(\eta_{k}\right\lrcorner *_{\varphi} \varphi\right) \otimes \eta_{k},
$$

for any local orthonormal positively oriented frame field $\left(\eta_{k}\right)_{k=1}^{7}$ on $M$ G, p. 1217].

Proposition 16 (cf. [McL, §5]). A 3-dimensional submanifold $Y$, with some choice of orientation, is associative if and only if $\left.\chi\right|_{Y}=0$.

Suppose that $Y$ is a closed associative submanifold in a $G_{2}$-manifold $(M, \varphi)$ and let $\mathbf{v} \in \Gamma\left(N_{Y / M}\right)$ be a normal vector field along $Y$. The deformation map for $Y$ is defined as

$$
F: \mathbf{v} \in \Gamma\left(N_{Y / M}\right) \rightarrow \exp _{\mathbf{v}}^{*} \tau \in \Omega^{3}\left(Y,\left.T M\right|_{Y}\right)
$$

The linearization of $F$ at $\mathbf{v}=0$ is given by

$$
D \mathbf{v}=\sum_{i=1}^{3} e_{i} \times \nabla_{e_{i}}^{\perp} \mathbf{v},
$$

where $e_{1}, e_{2}, e_{3}$ is any positively oriented local orthonormal frame field of $T Y$ (thus $e_{3}=e_{1} \times e_{2}$ ), the cross-product is induced by $\varphi$ and a connection $\nabla^{\perp}$ on $N_{Y / M}$ is induced by the Levi-Civita connection of $(M, g(\varphi))$. Since $Y$ is 3-dimensional and associative, both $T Y$ and $N_{Y / M}$ are trivial vector bundles [JK, Remark 2.14] and the expression (9) is valid globally over $Y$. There is an invariant interpretation of $N_{Y / M}$ as a vector bundle associated with a principal Spin(4)-bundle over $Y$ via the tensor product of a spin representation and some other representation. Then $D$ becomes the respective Dirac type operator (meaning that the principal symbol of $D^{2}$ is $\sigma\left(D^{2}\right)(p, \xi)=\|\xi\|^{2}$, for all $p \in Y)$.

The map $F$ makes sense for an arbitrary $G_{2}$-structure $\varphi$ on $M$, but when the $G_{2}$-structure is not torsion-free the expression (9) for $D$ then has extra terms of order zero. So in this more general case $D$ is still a Dirac type operator with the same principal symbol.

We thus obtain. 


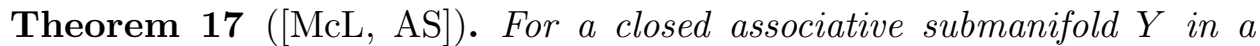
$G_{2}$-manifold $(M, \varphi)$, the Zariski tangent space to associative deformations of $Y$ is finite-dimensional, given by the kernel of the Dirac type operator $D$ in (91), an elliptic operator of index 0 . In particular, $Y$ is either rigid or the associative deformations of $Y$ are obstructed, i.e. a section $\mathbf{v}$ with $D \mathbf{v}=0$ need not arise as $\dot{s}_{0}$ from any 1-parameter family $Y_{t}=\exp s_{t}$ of associative submanifolds with $s_{0}=0$.

The deformations of compact associative submanifolds with boundary contained in a fixed submanifold (scaffold) were investigated by Gayet and Witt [GW], see also Gayet [G]. An appropriate choice of scaffold in this situation is given by a 4-dimensional submanifold $X$ such that no tangent space $T_{p} X$ contains associative 3 -planes. In particular, $X$ may be any coassociative submanifold and we shall assume this below for technical convenience. In this case, any associative calibrated submanifold with boundary in $X$ is volume minimizing in its relative homology class as $\left.\varphi\right|_{X}=0$ by Propositions 2(b) and 10.

Let $Y$ be a compact associative submanifold with boundary $\partial Y$ contained in a coassociative submanifold $X$. Denote by $\mathbf{n} \in \Gamma\left(\left.T Y\right|_{\partial Y}\right)$ the inward-pointing unit normal along $\partial Y$. For each point $p \in \partial Y$ the crossproduct of the $G_{2}$-structure $\varphi$

$$
J(v)=\mathbf{n}_{p} \times v
$$

defines an (orthogonal) complex structure on the orthogonal complement $\left(\mathbf{n}_{p}\right)^{\perp} \subset T_{p} M$. Further, $J$ acts on the fibres of the normal bundle $N_{\partial Y / X}$, making it into a complex line bundle. Note that $N_{\partial Y / X}$ is a subbundle of $\left.N_{Y / M}\right|_{\partial Y}$; the respective orthogonal complement $\mu_{\partial Y}$ is also invariant under $J$ and can be considered as a complex line bundle. The tangent spaces of $\partial Y$ are also preserved by $J$ and in this way $\partial Y$ is made into a compact Riemann surface. The latter complex line bundles satisfy an adjunction-type relation $\bar{\mu}_{\partial Y} \cong N_{\partial Y / X} \otimes_{\mathbb{C}} T \partial Y$ [GW, Lemma 3.2].

The infinitesimal associative deformation problem for $Y$ with boundary confined to $X$ can be expressed as

$$
D \mathbf{v}=0, \quad B\left(\left.\mathbf{v}\right|_{\partial Y}\right)=0, \quad \mathbf{v} \in \Gamma\left(N_{Y / M}\right),
$$

where $D$ is the Dirac type operator in (9) and the zero order operator $B$ is induced by the orthogonal projection $\left.N_{Y / M}\right|_{\partial Y} \rightarrow \mu_{\partial Y}$ with kernel $N_{\partial Y / X}$.

Gayet and Witt prove. 
Theorem 18 (GW, Thm. 4.4, Cor. 4.5]). Let $(M, \varphi)$ be a 7-manifold endowed with a $G_{2}$-structure and let $Y \subset M$ be a compact associative submanifold with boundary contained in a coassociative submanifold $Y \subset M$.

Then the linear operator $D \oplus B$ in (10) defines an elliptic boundary value problem with finite Fredholm index

$$
\operatorname{index}(D \oplus B)=\sum_{j}\left(\int_{\Sigma_{j}} c_{1}\left(N_{\Sigma_{j} / X}\right)+1-g_{j}\right) .
$$

Here $\Sigma_{j}$ denote the boundary components of $\partial Y$ with $g_{j}$ the genus of $\Sigma_{j}$, and $c_{1}\left(N_{\Sigma_{j} / X}\right)$ is the first Chern class of the complex line bundle $N_{\Sigma_{j} / X}$.

The key point in the proof of Theorem 18 is that the index in question can be computed as the index of the Cauchy-Riemann operator $\bar{\partial}_{\partial Y / X}$ associated with the complex structure $J$ on $N_{\partial Y / X}$.

Example 19 ([GW, p.2364]). When the associative submanifold has a boundary, the index in Theorem 18 can be positive. One simple example uses a construction by Bryant and Salamon [BS] of torsion-free $G_{2}$-structure $\varphi$ inducing a complete metric with holonomy $G_{2}$ on $\mathcal{S}=S^{3} \times \mathbb{R}^{4}$, the total space of the spinor bundle over the standard round 3 -sphere. The zero section $S^{3} \times\{0\}$ is an associative submanifold, being the fixed locus of the $G_{2}$-involution acting as -1 on the fibres (note [J, Prop. 12.3.7]). Take $Y \subset S^{3} \times\{0\}$ to be a 3 -dimensional ball, so $\partial Y=S^{2}$. Let $a$ be a nowhere vanishing section of $\left.\mathcal{S}\right|_{\partial Y}=\partial Y \times \mathbb{R}^{4} \rightarrow \partial Y$. Then $a, J a$ (with $J$ as defined above) generate a trivial complex line bundle, denote its total space by $\tilde{X}$. It can be checked that there is a local coassociative submanifold $X \subset \mathcal{S}$ containing $\partial Y$ and with $T_{p} X=T_{p} \tilde{X}$ at each $p \in \partial Y$. Theorem 18 then applies and the deformation problem has index 1 . This example generalizes, under additional assumptions, to a complex line bundle $\tilde{X}$ having positive degree $n$, then the respective index is $n+1$ [GW, p.2364].

Gayet and Witt also gave a generalization of Theorem 18 where $X$ is only required to contain no associative 3 -planes in its tangent spaces. The latter property is preserved under small perturbations of the $G_{2}$-structure. The deformation problem remains elliptic and the index formula (11) still holds (with appropriate modification of the definition of the boundary operator $B$ ). This does not guarantee a smooth moduli space of associative deformations even when the index of $D \oplus B$ is non-negative. However, Gayet [G. Thm. 1.4] proved that a smooth moduli space of dimension index $D \oplus B$ can be obtained by arbitrary small generic perturbation of the scaffold $X$. 


\section{Cayley submanifolds in Spin(7)-manifolds}

The calibration considered in this section is defined on 8-dimensional manifolds with a torsion-free Spin(7)-structure. We begin with a short summary of the Spin(7)-structure and the Cayley calibration and refer to [HL] and [J, Ch. 11,12] for further details. There is a certain, though only partial, analogy with the 'geometries' considered in the previous sections.

The group $\operatorname{Spin}(7)$ can be defined, following $[\mathrm{Br}$, as the stabilizer, in the standard action of $G L(8, \mathbb{R})$ on $\Lambda^{4}\left(\mathbb{R}^{8}\right)^{*}$, of the 4 -form $\Phi_{0}$ written in standard coordinates as

$$
\begin{aligned}
\Phi_{0} & =d x_{1234}+d x_{1256}+d x_{1278}+d x_{1357}-d x_{1368}-d x_{1458}-d x_{1467} \\
& -d x_{2358}-d x_{2367}-d x_{2457}+d x_{2468}+d x_{3456}+d x_{3478}+d x_{5678}
\end{aligned}
$$

where $\mathrm{d} x^{1234}=d x^{1} \wedge d x^{2} \wedge d x^{3} \wedge d x^{4}$ and so on. The form $\Phi_{0}$ arises by considering $\mathbb{R}^{8}$ as the (normed) algebra of octonions, or Cayley numbers, and setting $\Phi_{0}(x, y, z, w)=\frac{1}{2}\langle x(\bar{y} z)-z(\bar{y} x), w\rangle$. In this way, $\operatorname{Spin}(7)$ is also identified as a subgroup of $S O(8)$. This form is also self-dual $* \Phi_{0}=\Phi_{0}$ with respect to the standard Euclidean metric and orientation.

Given an oriented 8-manifold $M$, define a subbundle of 4 -forms $\mathcal{A} M \subset$ $\Lambda^{4} T^{*} M$ with the fibre $\mathcal{A}_{p} M$ at each $p \in M$ being the set of all 4 -forms that can be identified with $\Phi_{0}$ via an orientation-preserving isomorphism $T_{p} M \rightarrow$ $\mathbb{R}^{8}$. The fibres of $\mathcal{A} M$ are diffeomorphic to the orbit $G L_{+}(8, \mathbb{R}) / \operatorname{Spin}(7)$ of $\Phi_{0}$, a 43-dimensional submanifold of the 70 -dimensional vector space $\Lambda^{4}\left(\mathbb{R}^{8}\right)^{*}$.

A choice of 4 -form $\Phi \in \Gamma(\mathcal{A} M)$ is equivalent to a choice of a $\operatorname{Spin}(7)$ structure on $M$. By the inclusion $\operatorname{Spin}(7) \subset S O(8)$, every such $\Phi$ induces on $M$ a metric $g=g(\Phi)$, an orientation and a Hodge star $*_{\Phi}$, with $*_{\Phi} \Phi=\Phi$. We shall sometimes refer to $\Phi$ as a $\operatorname{Spin}(7)$-structure.

When a form $\Phi \in \mathcal{A} M$ is closed, $d \Phi=0$, we say that the $\operatorname{Spin}(7)$ structure $\Phi$ is torsion-free and that $(M, \Phi)$ is a $\operatorname{Spin}(7)$-manifold. The condition $d \Phi=0$ is equivalent to the metric $g(\Phi)$ being Ricci-flat with reduced holonomy contained in $\operatorname{Spin}(7)[\mathrm{Fe}$. In this case, $\Phi$ defines a calibration on $M$.

Given a $\operatorname{Spin}(7)$-structure on an 8-manifold $M$, we say that an oriented 4-dimensional submanifold $P \subset M$ is a Cayley submanifold if $\left.\Phi\right|_{P}=\operatorname{vol}_{P}$ We say that $P$ is a Cayley calibrated submanifold if in addition $d \Phi=0$, i.e. precisely if $P$ is calibrated by $\Phi$.

Let $P$ is a compact Cayley calibrated submanifold of a $\operatorname{Spin}(7)$-manifold $(M, \Phi)$. The infinitesimal Cayley deformations of $P$ are given by the kernel 
of a first order elliptic operator $D: \Gamma\left(N_{P / M}\right) \rightarrow \Gamma(E)$, for a rank 4 vector bundle $E$ over $P$,

$$
E=\left\{\left.\alpha \in \Lambda_{7}^{2} M\right|_{P}:\left.\alpha\right|_{T P}=0\right\} .
$$

Here $\Lambda_{7}^{2} M \subset \Lambda^{2} T^{*} M$ is a subbundle corresponding to an irreducible representation of $\operatorname{Spin}(7)$ on the space of 2 -forms $\Lambda^{2}\left(\mathbb{R}^{8}\right)^{*}$. The following result was proved by McLean.

Theorem 20 ([McL, Thm. 6.3]). Let $P$ be a Cayley submanifold of a Spin(7)-manifold $(M, \Phi)$. Then the Zariski tangent space to Cayley deformations of $P$ is finite-dimensional, given by the kernel of the elliptic operator

$$
D: \mathbf{v} \in \Gamma\left(N_{P / M}\right) \rightarrow \sum_{i=1}^{4} e_{i} \times \nabla_{e_{i}}^{\perp} \mathbf{v} \in \Gamma(E),
$$

where $e_{1}, e_{2}, e_{3}, e_{4}$ is any positively oriented local orthonormal frame field of TP, the cross-product is induced by the $\operatorname{Spin}(7)$-structure $\Phi$ and a connection $\nabla^{\perp}$ on $N_{P / M}$ is induced by the Levi-Civita connection of $(M, g(\Phi))$.

Remarks. When $P$ is a spin manifold, there is an invariant interpretation of $D$ using a spin structure on $P[\mathrm{McL}, \S 6]$. Denote by $\mathcal{S}_{+}$and $\mathcal{S}_{-}$the positive and negative spinor bundles over $P$. Then

$$
N_{P / M} \otimes_{\mathbb{R}} \mathbb{C} \cong \mathcal{S}_{+} \otimes_{\mathbb{C}} F \text { and } E \otimes_{\mathbb{R}} \mathbb{C} \cong \mathcal{S}_{-} \otimes_{\mathbb{C}} F,
$$

for some quaternionic line bundle $F$ over $P$. The operator $D$ is identified, via (14), with a positive Dirac type operator associated with a connection on $F$.

Theorem 20 was extended to arbitrary $\operatorname{Spin}(7)$-structures in [GIP, §13]. When the $\operatorname{Spin}(7)$-structure is not torsion-free, the expression (13) for $D$ has extra terms of order zero. This does not affect the principal symbol or the index of $D$.

Deformations of Cayley submanifolds were further investigated by Ohst and included the following.

Theorem 21 ([0, Prop. 3.4 and Thm. 3.10]). Let $(M, \Phi)$ be an 8-manifold with a Spin(7)-structure and $P \subset M$ a closed Cayley submanifold. Then

(a) the index of the operator (13) associated with $P$ is

$$
\text { index } D=\frac{1}{2} \chi(P)+\frac{1}{2} \sigma(P)-[P] \cdot[P],
$$

where $\chi(P)$ is the Euler characteristic, $\sigma(P)$ is the signature and $[P] \cdot[P]$ is the self-intersection number of $P$. 
(b) For every generic $\operatorname{Spin}(7)$-structure $\tilde{\Phi}$ on $M$ such that $\|\tilde{\Phi}-\Phi\|$ is sufficiently small and $\tilde{\Phi}$ induces the same metric $g(\tilde{\Phi})=g(\Phi)$ the following holds. The moduli space of Cayley submanifolds with respect to $\tilde{\Phi}$ which are $C^{1, \alpha}$-close to $P(0<\alpha<1)$ is either empty or a smooth manifold of dimension index $D$ (if index $D \geq 0$ ).

Remarks. The submanifold $P$ need not be Cayley with respect to $\tilde{\Phi}$, thus $P$ need not be in the respective moduli space. The norm $\|\tilde{\Phi}-\Phi\|$ can be taken to be the $C^{1, \alpha}$-norm on a compact neighbourhood of $P$.

The variant of Theorem 21 also holds with $\tilde{\Phi}$ generic in the set of all $\operatorname{Spin}(7)$-structures close to $\Phi$, i.e. without the restriction on the metric $g(\tilde{\Phi})$.

We next turn to deformations of compact Cayley submanifolds with boundary in a fixed submanifold (scaffold). The result given below is again due to Ohst and allows a range of dimensions of the scaffold.

Theorem 22 ([Q, Thm. 4.18]). Let $(M, \Phi)$ be a Spin(7)-manifold and $W$ a submanifold of $M$ with $3 \leq \operatorname{dim} W \leq 7$. Let $P$ be a compact, connected Cayley submanifold of $M$ with non-empty boundary $\partial P \subseteq W$ such that $P$ and $W$ meet orthogonally.

Then for every generic torsion-free Spin(7)-structure $\tilde{\Phi}$ which is $C^{2, \alpha}$ close to $\Phi$, the moduli space of all Cayley (calibrated) submanifolds in $(M, \tilde{\Phi})$ which are $C^{2, \alpha}$-close to $P$ and have boundary contained in $W$ and meet $W$ orthogonally in the metric $g(\tilde{\Phi})$ is a finite set (possibly empty). Here $0<\alpha<1$.

The proof of Theorem 22 uses a second order elliptic boundary problem implied by the linearization $D$ in (13) of the Cayley deformation map. This is because $D$ admits no suitable elliptic boundary conditions [O, Prop. 4.21].

The condition $\left.\Phi\right|_{W}=0$ required in Proposition 2(b) for the volumeminimizing property in the relative homology class of $P$ can only hold if $\operatorname{dim} W \leq 4$. Suppose that $\partial P$ is a deformation retract of $W$. If $\operatorname{dim} W=5$, then $v=d\left(*_{W}\left(\left.\Phi\right|_{W}\right)\right)^{b}$ restricts to a vector field on $\partial P$. If, further, $v$ is parallel with respect to the induced metric on $W$, then $P$ is volumeminimizing among the nearby deformations in its relative homology class.

When $\operatorname{dim} W=6$ and $d\left(*_{W}\left(\left.\Phi\right|_{W}\right)\right)=0$, the scaffold $W$ is a symplectic submanifold. Then $P$ minimizes the volume among all the submanifolds $P^{\prime}$ in its relative homology class, with boundary $\partial P^{\prime} \subset W$ a Lagrangian nearby deformation of $\partial P$. In all of the above situations, the minimal volume in the relative homology class of $P$ is attained precisely by Cayley calibrated submanifolds [O, §5.1]. 


\section{References}

[AS] S. Akbulut and S. Salur. Deformations in $G_{2}$ manifolds, $A d v$. Math. 217 (2008), 2130-2140.

[Br] R.L. Bryant. Metrics with exceptional holonomy. Ann. of Math. (2) 126 (1987), 525-576.

[BS] R.L. Bryant and S.M. Salamon. On the construction of some complete metrics with exceptional holonomy. Duke Math. J., 58 (1989), 829-850.

[Bu] A. Butscher. Deformations of minimal Lagrangian submanifolds with boundary. Proc. Amer. Math. Soc. 131 (2002), 1953-1964.

[CDGM] S. Cappell, D. DeTurck, H. Gluck, E. Y. Miller. Cohomology of harmonic forms on Riemannian manifolds with boundary. Forum Math. 18 (2006), 923-931.

[FG] M. Fernández and A. Gray. Riemannian manifolds with structure group $G_{2}$. Ann. Mat. Pura Appl. (4) 132 (1982), 19-45.

[Fe] M. Fernández. A classification of Riemannian manifolds with structure group Spin(7). Ann. Mat. Pura Appl. (4) 143 (1986), $101-122$.

[G] D. Gayet. Smooth moduli spaces of associative submanifolds. Quarterly J. Math., 65 (2014), 1213-1240.

[GW] D. Gayet and F. Witt. Deformations of associative submanifolds with boundary. Adv. Math. 226 (2011), 2351-2370.

[GIP] J. Gutowski, S. Ivanov and G. Papadopoulos. Deformations of generalized calibrations and compact non-Kähler manifolds with vanishing first Chern class. Asian J. Math. 7 (2003), 39-79.

$[\mathrm{H}] \quad$ R. Harvey. Spinors and calibrations. Academic Press Inc., Boston, 1990.

[HL] R. Harvey and H. B. Lawson. Calibrated geometries. Acta Math. 148 (1982), 47-152.

$[\mathrm{J}] \quad$ D.D. Joyce. Riemannian holonomy groups and calibrated geometry. OUP, Oxford, 2007. 
[JK] D.D. Joyce and S. Karigiannis. A new construction of compact torsion-free $G_{2}$-manifolds by gluing families of Eguchi-Hanson spaces. J. Diff. Geom., to appear. arXiv:1707.09325

[JS] D.D. Joyce and S. Salur. Deformations of asymptotically cylindrical coassociative submanifolds with fixed boundary. Geom. Topol. 9 (2005), 1115-1146.

[KL] A.G. Kovalev and J.D. Lotay. Deformations of compact coassociative 4-folds with boundary. J. Geom. Phys. 59 (2009) 63-73.

[L] S. Lang, Differential manifolds. Addison-Wesley, Reading, Massachusetts, 1972 .

[McL] R.C. McLean. Deformations of calibrated submanifolds. Comm. Anal. Geom. 6 (1998), 705-747.

[O] M. Ohst. Deformations of Cayley submanifolds. PhD Thesis, University of Cambridge, 2015. https://doi.org/10.17863/CAM.16246

[Sa] S. Salur. Deformations of special Lagrangian submanifolds. Commun. Contemp. Math. 2 (2000), 365-372.

[Sch] G. Schwarz. Hodge decomposition - a method for solving boundary value problems. Springer-Verlag, Berlin, 1995. 